\title{
Hard X-ray Nano-Holotomography of Formalin-Fixated and Paraffin-Embedded Human Brain Tissue
}

Anna Khimchenko ${ }^{1, \#}$, Alexandra Pacureanu ${ }^{2}$, Christos Bikis ${ }^{1}$, Simone E. Hieber ${ }^{1}$, Peter Thalmann ${ }^{1}$, Hans Deyhle ${ }^{1}$, Gabriel Schweighauser ${ }^{3}$, Jürgen Hench $^{3}$, Stephan Frank ${ }^{3}$, Magdalena Müller-Gerbl ${ }^{4}$, Georg Schulz ${ }^{1}$, Peter Cloetens ${ }^{2}$, and Bert Müller ${ }^{1, *}$

1. Biomaterials Science Center (BMC), DBE, University of Basel, Allschwil, Switzerland

2. European Synchrotron Radiation Facility (ESRF), Grenoble, France

3. Institute of Pathology, Basel University Hospital, Basel, Switzerland

${ }^{4 .}$ Musculoskeletal Research Group, DBM, University of Basel, Basel, Switzerland

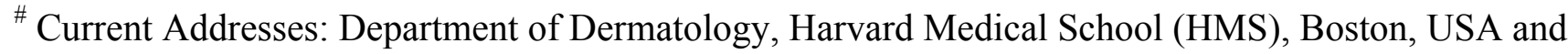

Wellman Center for Photomedicine, Massachusetts General Hospital (MGH), Boston, USA

*Corresponding author bert.mueller@unibas.ch

Scientists are fascinated by the complex micro- and nanostructure of the human brain, which has been visualized by means of modalities such as magnetic resonance, ultrasonic and optical imaging. X-raybased techniques, however, have hardly been considered because the conventional absorption-based Xray imaging provides only a weak contrast for physically soft tissues in general and brain tissues in particular, see e.g. [1]. Contrary to absorption modalities, X-ray phase contrast imaging is better suited for soft tissue visualization even resolving individual cells within the brain post mortem [2]. Today, we can image paraffin-embedded Purkinje cells even using advanced laboratory sources [3,4] and their subcellular structures including dendritic trees and nucleoli at synchrotron radiation facilities [5].

Recently, the X-ray nano-holotomography (XNH) has been established at the ID16A nano-imaging beamline, European Synchrotron Radiation Facility (Grenoble, France) [6] allowing hard X-ray tomography with a spatial resolution well below $100 \mathrm{~nm}$ [7].

The purpose of the study is the non-destructive three-dimensional imaging of volumes close to one cubic millimeter from formalin-fixated and paraffin-embedded human cerebellum with a spatial resolution beyond the optical limit. Brain specimens from donated bodies were treated according to established histology protocols, i.e. fixed in $4 \%$ histologically grade buffered formalin, dehydrated in ethanol, cleared in xylene and embedded in paraffin. Cylinders 0.5 to $0.7 \mathrm{~mm}$ in diameter were cut from the blocks. As the cylinders were larger than the field of view, local XNH measurements were performed. Four tomography datasets were acquired by placing the sample at preselected distances between the focus and the FReLoN detector. The high-brilliance coherent X-ray beam with a photon energy of $17 \mathrm{keV}$ was focused to a spot of $25 \mathrm{~nm} \times 37 \mathrm{~nm}$ by means of a pair of Kirkpatrick-Baez mirrors. Each set of radiographs for the given rotation angle was normalized with respect to the incoming beam, brought to the selected magnification, aligned and processed with an adapted contrast transfer function algorithm to determine the phase shift. To retrieve the local electron densities, we applied the filtered back projections using PyHST2. Subsequent to tomography, the block was sectioned to slides $4 \mu \mathrm{m}$ thin for histological examination. The software package VGStudio MAX 2.0 (Volume Graphics, Heidelberg, Germany) served for the segmentation and visualization. 
The multiscale approach began with a fast overview scan with an effective detector pixel length of $130 \mathrm{~nm}$. This scan supported the selection of subvolumes in the holotomography configuration, where pixel lengths down to $25 \mathrm{~nm}$ were used. Figure 1 represents tomography data acquired with an effective voxel length of $50 \mathrm{~nm}$. The data permit the imaging of individual Purkinje neurons including their subnuclear details and the semi-automated segmentation by intensity-based region-growing, see Figure 1. The granule and stellate cells have a distinctive electron density. Thus, the adaptive intensity thresholding is sufficient for automated quantification with $97 \%$ success.

We demonstrate that $\mathrm{XNH}$ non-destructively resolves the brain nanoanatomy ex vivo in 3D without staining. As there is a direct correlation between size, shape, or topology of neurons and brain disorders, $\mathrm{XNH}$ combined with sophisticated computational tools will support the diagnosis and improved understanding of brain pathologies.

References:

[1] M Germann et al, J. Neurosci. Meth. 170 (2008) 149

[2] G Schulz et al, J. Roy. Soc. Interface 7 (2010) 1665.

[3] A Khimchenko et al, NeuroImage 139 (2016) 26.

[4] M Töpperwien et al, Sci. Rep. 7 (2017) 42847.

[5] SE Hieber et al, Sci. Rep. 6 (2016) 32156.

[6] JC Da Silva et al, Optica 4 (2017) 492

[7] A Khimchenko et al, Adv. Sci. 5 (2018) 1700697; doi: 10.1002/advs.201700694.

[8] The authors acknowledge funding from the Swiss National Science Foundation projects 147172, 144535 and 133802. The beamtime was granted by the ESRF, proposal MD-969.
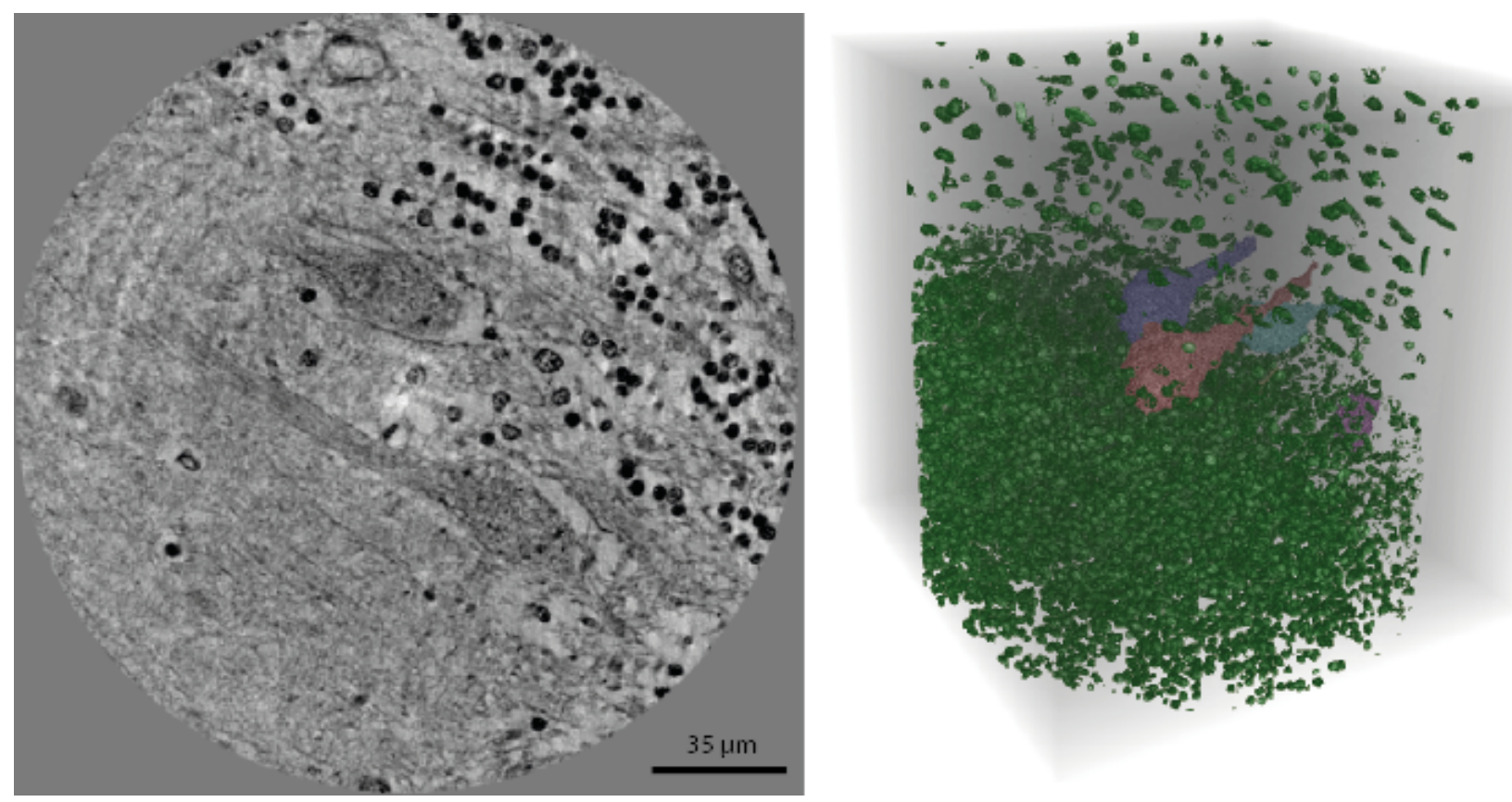

Figure. 1. $50 \mathrm{~nm}$-thin tomography slice of human cerebellum showing two Purkinje cells in its center and a three-dimensional representation of Purkinje (light colors) and other neurons (green color). 